Article

\title{
Multi-Oriented Object Detection in High-Resolution Remote Sensing Imagery Based on Convolutional Neural Networks with Adaptive Object Orientation Features
}

\author{
Zhipeng Dong ${ }^{1}$, Mi Wang ${ }^{2,3, *}$, Yanli Wang ${ }^{4}$, Yanxiong Liu ${ }^{1,5}\left(\mathbb{D}\right.$, Yikai Feng ${ }^{1,5}$ and Wenxue Xu ${ }^{1}(\mathbb{D})$ \\ 1 The First Institute of Oceanography, Ministry of Natural Resources, Qingdao 266061, China; \\ zhipengdong@fio.org.cn (Z.D.); yxliu@fio.org.cn (Y.L.); ykfeng@fio.org.cn (Y.F.); xuwx@fio.org.cn (W.X.) \\ 2 The Key Laboratory of Aerospace Information Security and Trusted Computing, Ministry of Education, \\ School of Cyber Science and Engineering, Wuhan University, Wuhan 430072, China \\ 3 The State Key Laboratory of Information Engineering in Surveying, Mapping, and Remote Sensing, \\ Wuhan University, Wuhan 430079, China \\ 4 The College of Geodesy and Geomatics, Shandong University of Science and Technology, Qingdao 266590, \\ China; wangyanli@sdust.edu.cn \\ 5 The Key Laboratory of Ocean Geomatics, Ministry of Natural Resources, Qingdao 266590, China \\ * Correspondence: wangmi@whu.edu.cn
}

check for updates

Citation: Dong, Z.; Wang, M.; Wang,

Y.; Liu, Y.; Feng, Y.; Xu, W.

Multi-Oriented Object Detection in

High-Resolution Remote Sensing

Imagery Based on Convolutional

Neural Networks with Adaptive

Object Orientation Features. Remote

Sens. 2022, 14, 950. https://doi.org/

$10.3390 /$ rs 14040950

Academic Editor: Lionel Bombrun

Received: 21 January 2022

Accepted: 14 February 2022

Published: 16 February 2022

Publisher's Note: MDPI stays neutral with regard to jurisdictional claims in published maps and institutional affiliations.

Copyright: (C) 2022 by the authors. Licensee MDPI, Basel, Switzerland. This article is an open access article distributed under the terms and conditions of the Creative Commons Attribution (CC BY) license (https:// creativecommons.org/licenses/by/ $4.0 /)$.

\begin{abstract}
In high-resolution earth observation systems, object detection in high spatial resolution remote sensing images (HSRIs) is the key technology for automatic extraction, analysis and understanding of image information. With respect to the multi-angle features of object orientation in HSRIs object detection, this paper presents a novel HSRIs object detection method based on convolutional neural networks (CNN) with adaptive object orientation features. First, an adaptive object orientation regression method is proposed to obtain object regions in any direction. In the adaptive object orientation regression method, five coordinate parameters are used to regress the object region with any direction. Then, a CNN framework for object detection of HSRIs is designed using the adaptive object orientation regression method. Using multiple object detection datasets, the proposed method is compared with some state-of-the-art object detection methods. The experimental results show that the proposed method can more accurately detect objects with large aspect ratios and densely distributed objects than some state-of-the-art object detection methods using a horizontal bounding box, and obtain better object detection results for HSRIs.
\end{abstract}

Keywords: high spatial resolution remote sensing image; convolutional neural network; object detection; adaptive object orientation features; deep learning

\section{Introduction}

In high-resolution earth observation systems, object detection in high spatial resolution remote sensing images (HSRIs) is the key technology for automatic extraction, analysis and understanding of image information [1-3]. It also plays an important role in the application of high-resolution earth observation systems to ocean monitoring, precision strike and military reconnaissance [4-6]. Object detection for HSRIs refers to the process of determining whether there are objects of interest and locating the objects of interest in the image [7]. In this paper, the objects detected are artificial geographical objects (e.g., storage-tanks, cars or airplanes) that have a clear boundary and have nothing to do with the HSRI background.

For object detection of HSRIs, scholars have carried out a lot of research. Mostly object detection methods usually use a three-stage mode of (1) extracting object candidate regions, (2) obtaining the features of object candidate regions, and (3) classifying object candidate regions using the features to detect objects in HSRIs [7]. For example, Xiao et al. [8] extracted object candidate regions using slide windows in various scales. Then, 
histograms of oriented gradients (HOG) of the object candidate regions were obtained. Finally, the object candidate regions were classified using support vector machines (SVM) to realize airplane and car detection in HSRIs. Cheng et al. [9] extracted object candidate regions using a slide window. Then, the HOG feature pyramid of the object candidate regions was obtained. Finally, according to the HOG feature pyramid, the object candidate regions were classified using SVM to detect airplanes in HSRIs. Diao et al. [10] used visual saliency to extract object candidate regions. Then, the features of object candidate regions were obtained and classified using deep belief networks to realize airplanes detection in HSRIs. Han et al. [11] extracted object candidate regions based on saliency map and visual attention computational model. Then, the features of object candidate regions were obtained and classified using fisher discrimination dictionary learning to realize multi-class object detection in HSRIs. The three-stage mode can achieve acceptable object detection results for object detection tasks in specific scenes [7]. However, remote sensing satellites in near-earth orbit acquire a large number of HSRIs from a top-down perspective every day, which is a way that is susceptible to illumination and weather conditions [12]. The three-stage mode cannot be effectively applied to detect objects of a large number of images in different complex scenes [3]. The universality and robustness of this mode are poor.

In recent years, many studies on deep learning have been carried out. The convolutional neural network (CNN) model is the most widely used deep learning model [13-22]. CNN does not need to use artificially designed features and can learn and extract effective features of the image using massive images and annotations. Moreover, with sufficient training data, $\mathrm{CNN}$ has good generalization ability, and can also maintain good universality and robustness in different complex scenes [3]. Therefore, the object detection methods based on CNN have been widely adopted to detect objects for HSRIs [23-27]. Long et al. [28] used regional CNN (R-CNN) to detect multi-class objects for HSRIs. Han et al. [12] applied transfer learning and Faster R-CNN to detect multi-class objects in HSRIs. Guo et al. [29] proposed a multi-scale CNN to detect objects in HSRIs, and obtained acceptable object detection results for HSRIs using an object detection framework based on multi-scale CNN. Chen et al. [30] applied a single shot multi-box detector (SSD) to detect airplanes of HSRIs. $\mathrm{Li}$ et al. [31] proposed an object detection framework based on CNN for object detection of remote sensing images. In the framework, there are two networks: region proposal network (RPN) and local-contextual feature fusion network. In the region proposal network (RPN), multiangle anchors were added. The double-channel feature fusion network was used to learn local and contextual properties. Deng et al. [32] proposed an object detection framework based on CNN for simultaneously detecting multi-class objects in remote sensing images with large scales variability. There were two subnetworks in the object detection framework. The object-like regions were generated in different scale layers using a multi-scale object proposal network. The object-like regions were classed based on fused feature maps using an accurate object detection network. Dong et al. [33] proposed an object detection framework based on CNN with suitable object scale features (CNN-SOSF) for multi-class object detection in HSRIs. CNN-SOSF provided acceptable multi-class object detection results in HSRIs. Liu et al. [34] designed an effective multiclass objects detection framework based on You Only Look Once version 2 (YOLOv2) to obtain acceptable object detection results for HSRIs. Ma et al. [35] proposed the improved YOLOv3 framework to detect collapsed buildings in HSRIs. However, in the above literature object detection frameworks based on CNN, all use the horizontal bounding box to detect objects in HSRIs, they are difficult to adapt to the densely packed object detection with a large aspect ratio, as shown in Figure 1a. In Figure 1a, there is a missed detection for the adjacent ships with a large aspect ratio. There is a large redundant area in the detection result of the ship. Therefore, for the objects detection of HSRIs, the object detection framework based on CNN needs to use the oriented bounding box (OBB) to improve the detection accuracy of the densely packed objects with a large aspect ratio, as shown in Figure 1b. 


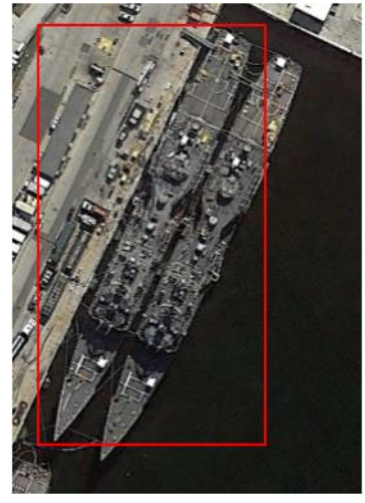

(a)

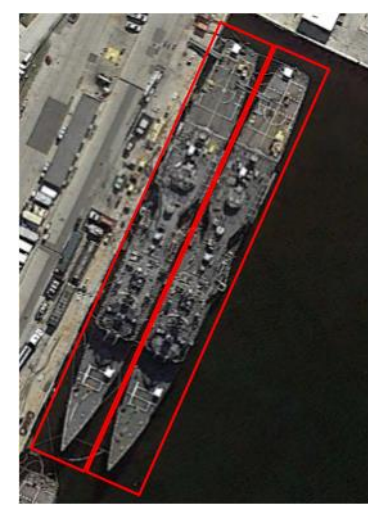

(b)

Figure 1. (a) Horizontal bounding box. (b) Oriented bounding box.

For using OBB to detect objects in HSRIs, some studies have been carried out. For example, Ding et al. [36] designed a Rotated Region of Interests (RRoI) learner to transform a Horizontal Region of Interest (HRoI) into an RRoI. The designed RRoI transformer was embedded into an object detector for orient object detection. Li et al. [37] proposed a featureattentioned object detection framework to detect orient objects in HSRIs. The proposed framework consisted of three components: feature-attentioned feature pyramid networks, multiple receptive fields attention-based RPN, and proposal-level attention-based ROI module. Yang et al. [38] proposed a multi-category rotation detector for small, cluttered and rotated objects. In the rotated detector, the supervised pixel attention network and the channel attention network were jointly explored for small and cluttered object detection by suppressing the noise and highlighting the object's features. For more accurate rotation estimation, the IOU constant factor was added to smooth L1 loss to address the boundary problem for the rotating bounding box. Wang et al. [39] provided a semantic attentionbased mask oriented bounding box representation for multi-category object detection for HSRIs. In the proposed oriented object detector, an inception lateral connection network was used to enhance the FPN. Furthermore, a semantic attention network was adopted to provide the semantic feature to help distinguish the object of interest from the cluttered background effectively. Compared with $\mathrm{HBB}$, object detectors based on OBB are more suitable for object detection of HSRIs. Therefore, object detection based on OBB has become a research hotspot.

With respect to the multi-angle features of object orientation in HSRIs object detection, this paper presents a novel HSRIs object detection method based on CNN with adaptive object orientation features (CNN-AOOF). First, an adaptive object orientation regression method is proposed. Then, a CNN framework for object detection in HSRIs is designed using the adaptive object orientation regression method.

The main contributions of this paper are as follows:

1. An HSRI object detection dataset with OBB, WHU-RSONE-OBB, is established and published to promote the development of object detection for HSRIs.

2. An adaptive object orientation regression method is proposed to obtain object regions in any direction.

3. An object detection framework based on $\mathrm{CNN}$ with adaptive object orientation features is designed to detect various objects for HSRIs.

4. The proposed method can more accurately detect objects with large aspect ratios and densely distributed objects than object detectors using a horizontal bounding box.

The rest of this paper is organized as follows. In Section 2, the CNN-AOOF framework is introduced in detail. In Sections 3 and 4, the datasets are described, and experimental results are discussed and analyzed. In Section 5, the experimental results are summarized, and the conclusions are drawn. 


\section{Materials and Methods}

In this paper, the CNN-AOOF framework is obtained using two steps. First, the adaptive object orientation regression method is proposed. Second, the CNN-AOOF framework is designed using the adaptive object orientation regression method.

\subsection{The Adaptive Object Orientation Regression Method}

At present, single-stage object detectors (such as YOLO [17], YOLOv2 [20] and SSD [18]) and two-stage object detectors (such as Fast-RCNN [15] and Faster-RCNN [16]) use four parameters $(x, y, w, h)$ to train and regress the coordinates of the object region. $x$ and $y$ are the coordinates of the center point of the object region. $w$ and $h$ are the width and height of the object region. The object region that is trained and regressed using four parameters $(x, y, w, h)$ is the horizontal bounding box, which is difficult to effectively couple the object region in HSRIs, as shown in Figure 1a. In order to well couple the object region in HSRIs, the adaptive object orientation regression method is proposed in this paper.

In the adaptive object orientation regression method, five parameters $(x, y, w, h, \theta)$ are used to train and regress the object region, as shown in Figure 2a. $x$ and $y$ are the coordinates of the center point of the object region. $w$ and $h$ are the width and height of the object region. In remote sensing image processing, the upper left corner is the coordinate origin $(0,0)$, the horizontal axis is the $X$ axis, and the vertical axis is the $Y$ axis, as shown in Figure 3. $\theta$ represents the angle of the clockwise rotation from the $X$ axis to the $Y$ axis. Therefore, in the adaptive object orientation regression method, $\theta$ is the angle between the corner point with the smallest $y$ value in the four corner point coordinates of the object region and the $X$ axis. The value range of $\theta$ is $(0, \pi / 2]$. When $\theta$ is $\pi / 2, \theta$ is the angle between the point $\mathrm{P}_{1}$ and the $\mathrm{X}$ axis, as shown in Figure $2 \mathrm{~b}$. $w$ and $h$ are the length of $\left|\mathrm{P}_{3} \mathrm{P}_{4}\right|$ and $\left|\mathrm{P}_{4} \mathrm{P}_{1}\right|$, respectively. The object region that is trained and regressed using five parameters $(x, y, w, h, \theta)$ is an arbitrary-oriented bounding box, which can well couple the object region in HSRIs.

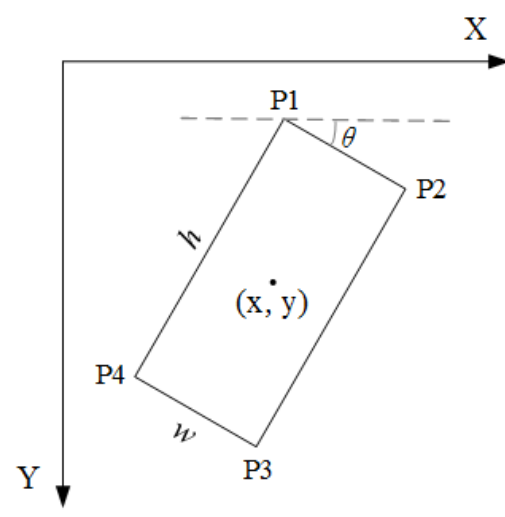

(a)

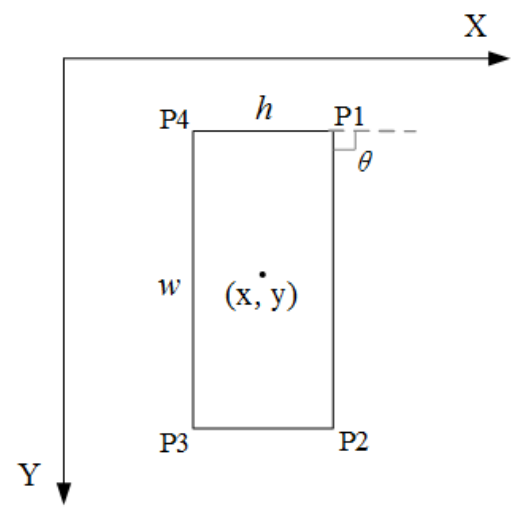

(b)

Figure 2. (a) Oriented bounding box using five parameters $(x, y, w, h, \theta)$. (b) Oriented bounding box with $\theta$ of $\pi / 2$.

In the adaptive object orientation regression method, five parameters $(x, y, w, h, \theta)$ of the object region are trained and regressed based on the anchor, as shown in Figure 4 . In Figure 4 , the dotted rectangle is the anchor at the position $(i, j)$ of the output feature map. In the process of training and regressing the object region, five parameters $(x, y, w, h, \theta)$ of the object region at the position $(i, j)$ are calculated as follows:

$$
\begin{aligned}
& O_{x}=i+\frac{1}{1+e^{-x_{0}}} \\
& O_{y}=j+\frac{1}{1+e^{-x_{1}}}
\end{aligned}
$$




$$
\begin{gathered}
O_{w}=a_{w} e^{x_{2}} \\
O_{h}=a_{h} e^{x_{3}} \\
O_{\theta}=\frac{\pi}{2} \times \frac{1}{1+e^{-x_{4}}}
\end{gathered}
$$

where $\left(O_{x}, O_{y}, O_{w}, O_{h}, O_{\theta}\right)$ are the regressed five parameters $(x, y, w, h, \theta)$ of the object region. $a_{w}$ and $a_{h}$ are $w$ and $h$ of the anchor, respectively, as shown in Figure 4 . $\left(x_{0}, x_{1}, x_{2}, x_{3}, x_{4}\right)$ are the output values of CNN correspond to the anchor at the position $(i, j)$ of the output feature map for regressing the coordinates of the object region, as shown in Figure 5. In Figure 5, at each position of the output feature map of 13 pixels $\times 13$ pixels, three object regions are trained and regressed based on the anchor. At each position of the output feature map, there are $3 \times(5+1+$ class number $) x$ outputs. The first five $x$ of each anchor are used to calculate $\left(O_{x}, O_{y}, O_{w}, O_{h}, O_{\theta}\right)$.

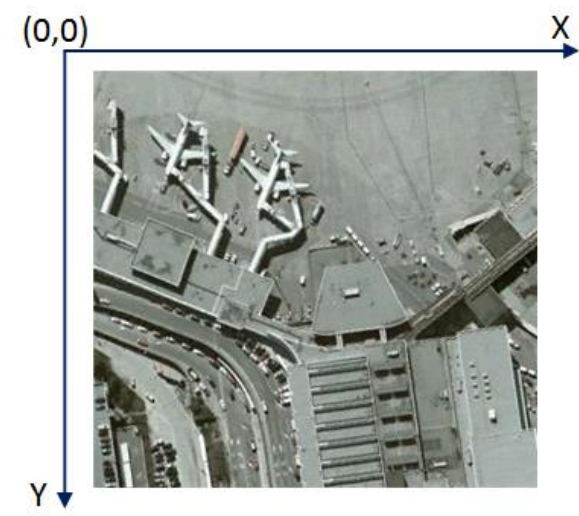

Figure 3. The coordinate system of remote sensing image processing.

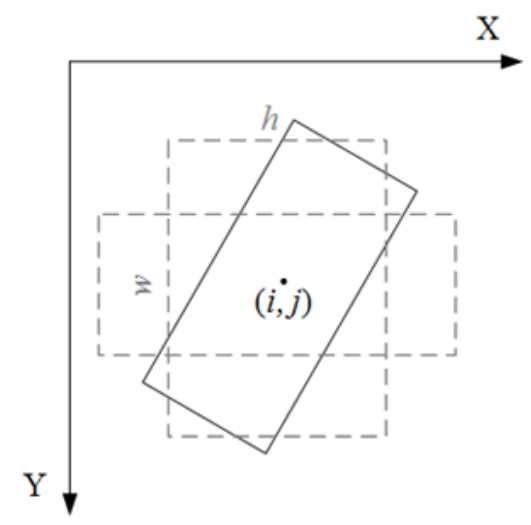

Figure 4. Coordinate regression of the object region based on the anchor.

In the adaptive object orientation regression method, the four corner point coordinates of the object region can be obtained using five parameters $(x, y, w, h, \theta)$ of the object region. The oriented bounding box can be drawn using the four corner point coordinates of the object region. The calculation formula is as follows:

$$
\left\{\begin{array}{l}
\left(x_{P 1}, y_{P 1}\right)=\left(x+\frac{h \sin \theta-w \cos \theta}{2}, y-\frac{h \sin \theta+w \cos \theta}{2}\right) \\
\left(x_{P 2}, y_{P 2}\right)=\left(x+\frac{h \sin \theta+w \cos \theta}{2}, y+\frac{h \sin \theta-w \cos \theta}{2}\right) \\
\left(x_{P 3}, y_{P 3}\right)=\left(x+\frac{h \sin \theta-w \cos \theta}{2}, y+\frac{h \sin \theta+w \cos \theta}{2}\right) \\
\left(x_{P 4}, y_{P 4}\right)=\left(x-\frac{h \sin \theta+w \cos \theta}{2}, y+\frac{h \sin \theta-w \cos \theta}{2}\right)
\end{array}\right.
$$


where $\left(x_{P 1}, y_{P 1}\right),\left(x_{P 2}, y_{P 2}\right),\left(x_{P 3}, y_{P 3}\right)$ and $\left(x_{P 4}, y_{P 4}\right)$ are the coordinates of the four points $P_{1}, P_{2}, P_{3}$ and $P_{4}$ of the object region, respectively.

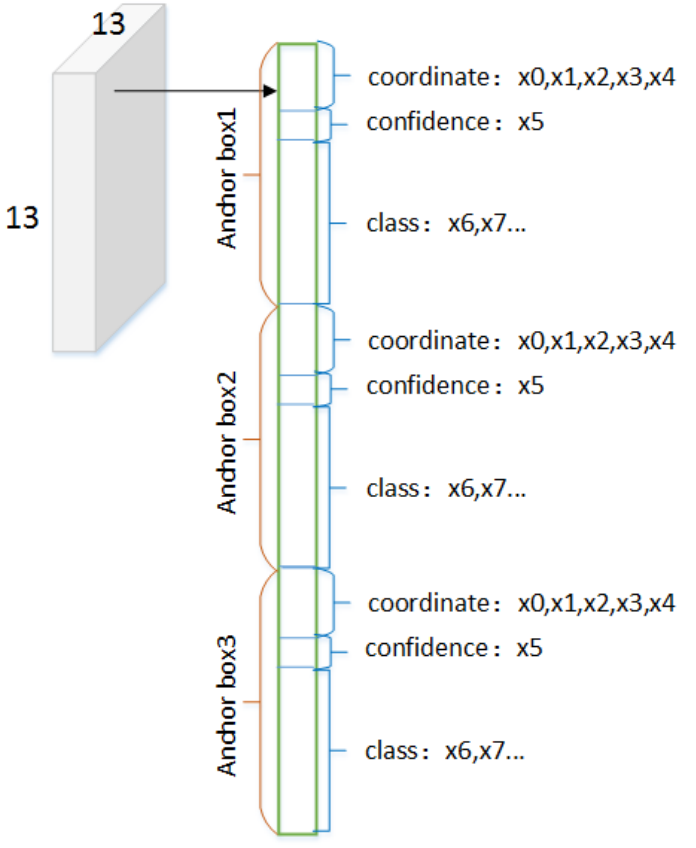

Figure 5. The output values of $\mathrm{CNN}$.

\subsection{CNN-AOOF Framework Design}

Using the adaptive object orientation regression method, a novel object detection framework based on CNN-AOOF for object detection in HSRIs is proposed. The CNNAOOF framework is a single-stage object detector, as shown in Figure 6. In the CNN-AOOF framework, darknet-53 [21] is used to generate the feature maps. The size of the input image in darknet-53 is 416 pixels $\times 416$ pixels. In the CNN-AOOF framework, the object region is trained and regressed based on the anchor on three different scale feature maps. On the feature map with the size of 13 pixels $\times 13$ pixels, object candidate regions are trained and regressed based on three anchors at each position of the feature map. The size of the three anchors are 116 pixels $\times 90$ pixels, 156 pixels $\times 198$ pixels, and 373 pixels $\times$ 326 pixels, respectively, as shown in the blue dotted rectangle in Figure 6 . The feature map with the size of 13 pixels $\times 13$ pixels is upsampled and combined with the feature map with the size of 26 pixels $\times 26$ pixels to form a new feature map with the size of 26 pixels $\times$ 26 pixels. On the new feature map with the size of 26 pixels $\times 26$ pixels, object candidate regions are trained and regressed based on three anchors at each position of the feature map. The size of the three anchors are 30 pixels $\times 61$ pixels, 62 pixels $\times 45$ pixels, and 59 pixels $\times 119$ pixels, respectively, as shown in the green dotted rectangle in Figure 6 . The new feature map with the size of 26 pixels $\times 26$ pixels is upsampled and combined with the feature map with the size of 52 pixels $\times 52$ pixels to form a new feature map with the size of 52 pixels $\times 52$ pixels. On the new feature map with the size of 52 pixels $\times 52$ pixels, object candidate regions are trained and regressed based on three anchors at each position of the feature map. The size of the three anchors are 10 pixels $\times 13$ pixels, 16 pixels $\times 30$ pixels, and 33 pixels $\times 23$ pixels, respectively, as shown in the red dotted rectangle in Figure 6 . 


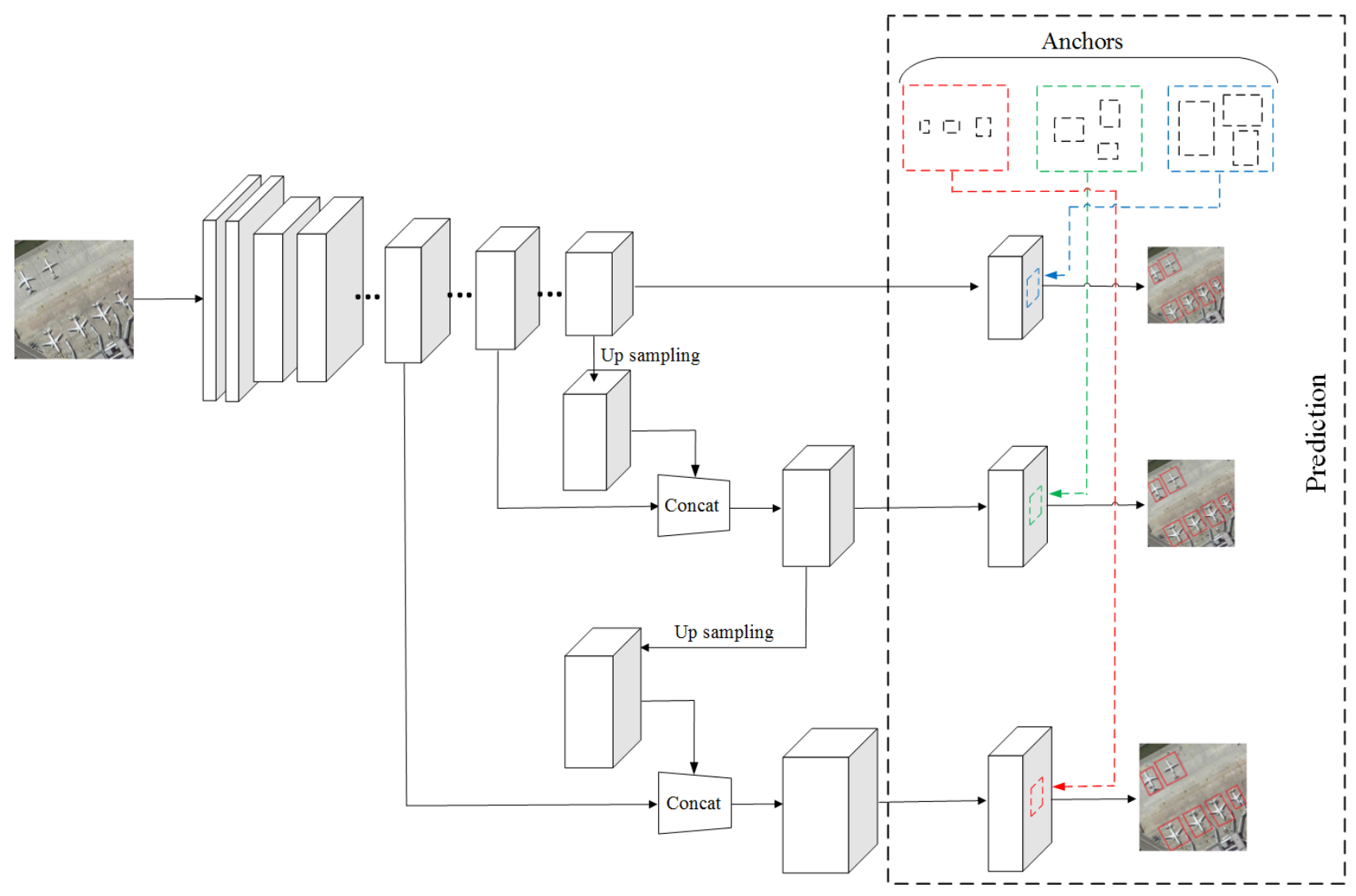

Figure 6. The object detection framework based on convolutional neural networks with suitable object scale features.

In the $\mathrm{CNN}-\mathrm{AOOF}$ framework, a multi-scale training method is used. Three object candidate regions are generated based on three anchors at each position of three different scale feature maps. If the intersection-over-union (IOU) overlap of the anchor and a ground truth box is the greatest among that of all anchors and a ground truth box, a positive label is assigned to the anchor. If a positive label is not assigned to the anchor, a negative label will be assigned to the anchor. In training the CNN-AOOF framework, there are $m \times m \times n \times(r+6)$ predicted value outputs on each feature map. The loss function of the $\mathrm{CNN}-\mathrm{AOOF}$ framework is calculated as follows:

$$
\begin{aligned}
& \text { Loss }=L_{\text {coord }}+L_{\text {class }}+L_{o b j} \\
& L_{\text {coord }}=\sum_{i}^{m} \sum_{j}^{m} \sum_{k}^{\mathrm{n}} I_{i j k}^{o b j}\left(2-w_{i j} \times h_{i j}\right)^{2}\left[\begin{array}{l}
\left(x_{i j}-i-s\left(x_{i j}^{*}\right)\right)^{2}+\left(y_{i j}-j-s\left(y_{i j}^{*}\right)\right)^{2} \\
+\left(\ln \left(\frac{w_{i j}}{w_{a}}\right)-w_{i j}^{*}\right)^{2}+\left(\ln \left(\frac{h_{i j}}{h_{a}}\right)-h_{i j}^{*}\right)^{2} \\
+\left(\frac{2 \theta_{i j}}{\pi}-s\left(\theta_{i j}^{*}\right)\right)^{2}
\end{array}\right] \\
& L_{\text {class }}= \begin{cases}\sum_{i}^{m} \sum_{j}^{m} \sum_{k}^{n} \sum_{l}^{r} I_{i j k}^{o b j} & l=\text { truth }_{\text {class }} \\
\sum_{i}^{m} \sum_{i}^{m} \sum_{k}^{n} \sum_{l}^{r} I_{i j k}^{o b j}\left(0-s\left(c_{i j l}^{*}\right)\right) & l \neq \text { truth }_{\text {class }}\end{cases} \\
& L_{o b j}= \begin{cases}\sum_{i}^{m} \sum_{j}^{m} \sum_{k}^{n} 1 & I_{i j k}^{o b j}=1 \\
\sum_{i}^{m} \sum_{j}^{m} \sum_{k}^{n}\left(0-s\left(u_{i j k}^{*}\right)\right)^{2} & I_{i j k}^{o b j} \neq 1\end{cases}
\end{aligned}
$$




$$
S(x)=\frac{1}{1+e^{-x}}
$$

where Loss is the training loss of CNN-AOOF framework. $L_{c o o r d}, L_{c l a s s}$ and $L_{o b j}$ are the training loss of coordinates, class and confidence of generated object regions based on anchors, respectively. $m$ is the width and height of the feature map. $n$ is the number of anchors at each position of the feature map. $I_{i j k}^{o b j}$ indicates whether a positive label is assigned to label $k$ anchor at position $(i, j)$ of the feature map. If a positive label is assigned to label $k$ anchor, $I_{i j k}^{o b j}$ is 1 , otherwise $I_{i j k}^{o b j}$ is $0 . w_{i j}$ and $h_{i j}$ are the width and height of the ground truth box corresponding to the label $k$ anchor at position $(i, j)$ of the feature map, respectively. $\left(x_{i j}, y_{i j}, w_{i j}, h_{i j}, \theta_{i j}\right)$ are the five parameters of the ground truth box. $\left(x_{i j}^{*}, y_{i j}^{*}, w_{i j}^{*}, h_{i j}^{*}, \theta_{i j}^{*}\right)$ are the framework output values to calculate five parameters of the generated object region based on label $k$ anchor. $w_{a}$ and $h_{a}$ are the width and height of the label $k$ anchor, respectively. $r$ is the classification number of the object. $c_{i j l}^{*}$ is the framework output value of different classifications of the generated object region based on label $k$ anchor. $u_{i j k}^{*}$ is the framework output value of the object confidence of generated object region based on label $k$.

In the testing process of the CNN-AOOF framework, all $x, y, \theta$, confidence and classification values among all the output values of the $\mathrm{CNN}-\mathrm{AOOF}$ framework are processed using Formula (11). Then the five parameters of the generated object region based on the anchor at each position of the feature map are obtained using Formulas (1)-(5). If the confidence of the generated object region is greater than the threshold, it is retained, otherwise, it is removed. The confidence threshold of various object detection results of CNN-AOOF is set to 0.05 for quantitative evaluation. The classification of retained generated object regions is determined based on classification output values of the CNN-AOOF framework. To reduce redundancy, the non-maximum suppression (NMS) algorithm is applied to retained generated object regions based on their confidence. The IOU threshold is set to 0.3 in the NMS algorithm. After NMS, the object detection result of an HSRI is obtained.

\section{Results}

Some state-of-the-art object detection algorithms (Faster-RCNN, CNN-SOSF, YOLOv2 and YOLOv3) have been effectively applied to object detection for HSRIs. To examine the object detection effectiveness of CNN-AOOF, four HSRI object detection datasets (WHURSONE-OBB, UCAS-AOD, HRSC2016 and DOTA) are used to compare CNN-AOOF with Faster-RCNN, CNN-SOSF, YOLOv2 and YOLOv3. CNN-AOOF is based on the darknet framework and programmed using $\mathrm{C}++$. The experiments are carried out on a server with Inter(R) Xeon(R) CPU E5-2667 v4 @ 3.20 GHz, NVIDIA Quadro M4000 (8 GB GPU memory), 16 GB RAM, and Windows 10 operating system.

\subsection{Object Detection for WHU-RSONE-OBB}

Large-scale object detection datasets are the basis and key for supporting object detection methods based on $\mathrm{CNN}$ to achieve high performance [40,41]. Therefore, an object detection dataset with OBB for HSRI, WHU-RSONE-OBB, is established and made publication to promote the development of HSRI object detection in this paper. In WHU-RSONEOBB, images were obtained from SuperView1 images, Tianditu, and Google Earth images. In WHU-RSONE-OBB, there are 5977 images. The size of images in WHU-RSONE-OBB ranges from 600 pixels $\times 600$ pixels to 1372 pixels $\times 1024$ pixels. The spatial resolution of images ranges from $0.5 \mathrm{~m}$ to $0.8 \mathrm{~m}$. There are three kinds of geospatial objects (airplane, storage-tank and ship) in WHU-RSONE-OBB, and object samples are labeled using OBB. The number of the three kinds of geospatial objects in WHU-RSONE-OBB is shown in Table 1. 
Table 1. The number of three kinds of objects in WHU-RSONE-OBB.

\begin{tabular}{cc}
\hline Object & Number \\
\hline Airplane & 15,703 \\
Storage-tank & 24,692 \\
Ship & 10,263 \\
\hline
\end{tabular}

In this paper, mean average precision (mAP) is used as the evaluation criteria for object detection results of object detectors [42]. If the IOU of the bounding box of object detection result and bounding box of ground-truth is equal to or greater than 0.5 , the object detection result is considered correct, and vice versa. The larger the mAP value is, the higher accuracy of the object detector becomes, and vice versa. The mAP is obtained using the following formula:

$$
m A P=\frac{1}{n} \sum_{i}^{n} A P_{i}
$$

where $i$ is the label of an object class. $n$ is the class number of the detected objects. $A P_{i}$ is the average precision of label $i$ class. Its value is the area under the precision-recall curve (PRC), as shown in Figure 7.

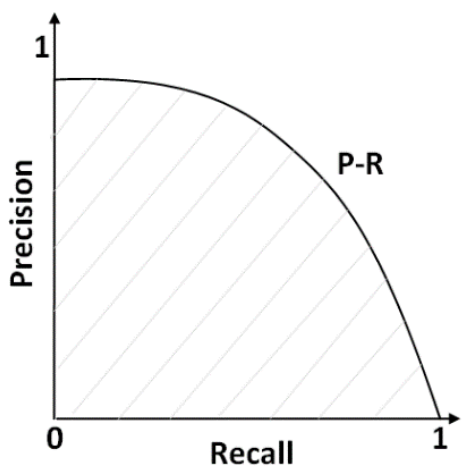

Figure 7. Precision-recall curve diagram.

In WHU-RSONE-OBB, we randomly select 4781 images as the training set, 598 images as the validation set, and 598 images as the testing set. Using WHU-RSONE-OBB, CNN$\mathrm{AOOF}$ and some state-of-the-art object detection algorithms are trained and tested. Table 2 shows the quantitative comparison results of $\mathrm{CNN}-\mathrm{AOOF}$ and some state-of-the-art object detection algorithms. In Table 2, the AP values of airplane, storage-tank and ship are 0.9857, 0.8831 and 0.792, respectively, in the object detection results using CNN-AOOF. The AP values of various objects using $\mathrm{CNN}-\mathrm{AOOF}$ are greater than those of other object detection algorithms. Moreover, the $\mathrm{mAP}$ value of $\mathrm{CNN}-\mathrm{AOOF}$ is the largest among the five object detection algorithms. These show that $\mathrm{CNN}-\mathrm{AOOF}$ can obtain more accurate object detection results than other object detection algorithms for HSRIs in WHU-RSONE-OBB.

Table 2. Performance comparisons of the five object detection algorithms in terms of AP values for WHU-RSONE-OBB dataset. The bold number represents the maximum value of each column.

\begin{tabular}{ccccc}
\hline & Airplane & Storage-Tank & Ship & mAP \\
\hline Faster-RCNN [16] & 0.9486 & 0.5634 & 0.7638 & 0.7586 \\
CNN-SOSF [33] & 0.9521 & 0.7461 & 0.7520 & 0.8167 \\
YOLOv2 [20] & 0.7116 & 0.3166 & 0.4422 & 0.4901 \\
YOLOv3 [21] & 0.9776 & 0.8709 & 0.7865 & 0.8784 \\
CNN-AOOF & $\mathbf{0 . 9 8 5 7}$ & $\mathbf{0 . 8 8 3 1}$ & $\mathbf{0 . 7 9 2 0}$ & $\mathbf{0 . 8 8 6 9}$ \\
\hline
\end{tabular}


Figure 8 shows the PRCs of the five object detection algorithms for object detection results of WHU-RSONE-OBB. In Figure 8, for airplane, storage-tank and ship, the PRC areas of $\mathrm{CNN}-\mathrm{AOOF}$ are greater than those of the other object detection algorithms. The experimental results show that $\mathrm{CNN}-\mathrm{AOOF}$ outperforms the other object detection algorithms, and can obtain more accurate object detection results.

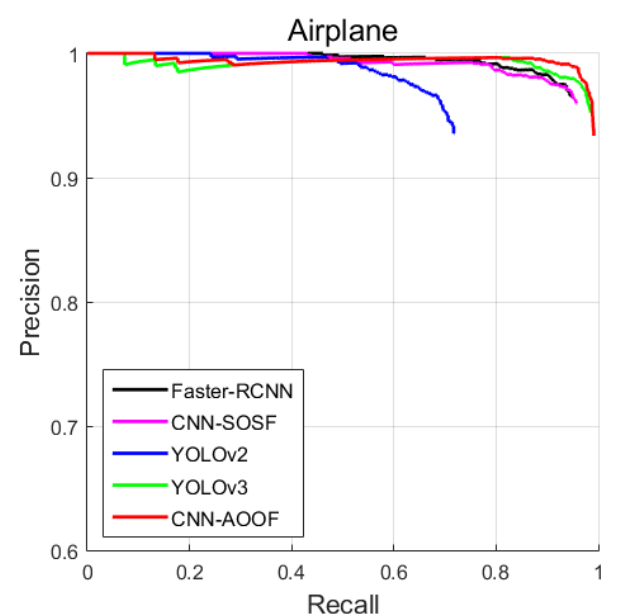

(a)

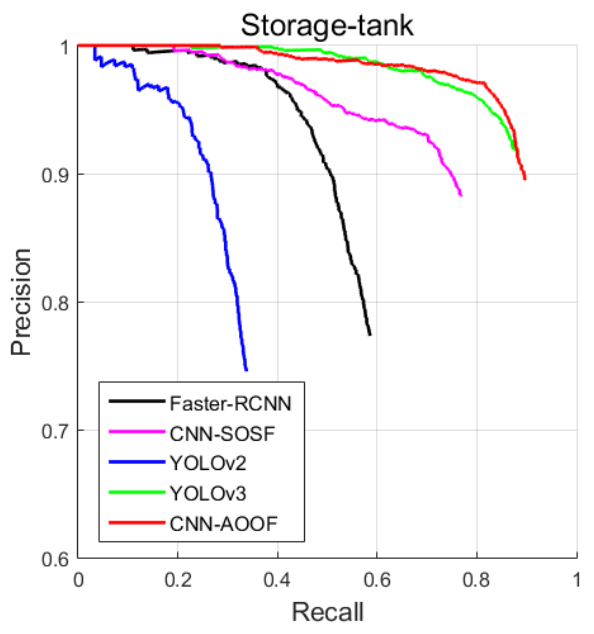

(b)

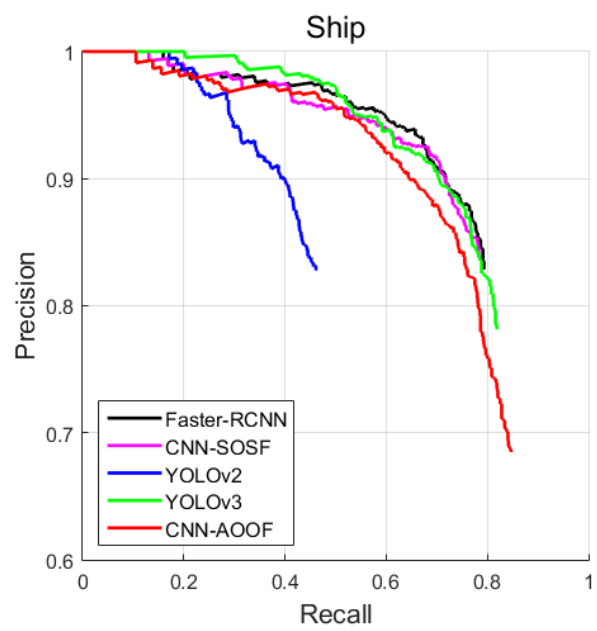

(c)

Figure 8. PRCs of the five object detection algorithms for (a) airplane, (b) storage-tank and (c) ship in WHU-RSONE-OBB.

Table 3 shows the average time consumptions of five object detection algorithms for per image object detection in WHU-RSONE-OBB. In Table 3, the average time consumptions of the five object detection algorithms are $0.467 \mathrm{~s}, 0.528 \mathrm{~s}, 0.102 \mathrm{~s}, 0.139 \mathrm{~s}$ and $0.233 \mathrm{~s}$, respectively. The average time consumptions of the five object detection algorithms are less than $1 \mathrm{~s}$. The experimental results show that the objects in HSRIs can be detected efficiently using CNN-AOOF.

Table 3. The average time consumptions of the five object detection algorithms.

\begin{tabular}{cc}
\hline & Time/Per Image (s) \\
\hline Faster-RCNN [16] & 0.467 \\
CNN-SOSF [33] & 0.528 \\
YOLOv2 [20] & 0.102 \\
YOLOv3 [21] & 0.139 \\
CNN-AOOF & 0.233 \\
\hline
\end{tabular}

\subsection{Object Detection for UCAS-AOD}

To further verify the object detection effectiveness of CNN-AOOF, UCAS-AOD [43] is used to compare CNN-AOOF with some state-of-the-art object detection algorithms (FasterRCNN, CNN-SOSF, YOLOv2 and YOLOv3). UCAS-AOD is an HSRI object detection dataset that contains two kinds of objects: airplane and car. Object samples are labeled using OBB. Images are cropped from Google Earth. There are 1510 images in UCAS-AOD, and the size of images ranges from 1280 pixels $\times 659$ pixels to 1372 pixels $\times 941$ pixels. In line with $[44,45]$, we randomly select 1060 images for training and 450 images for testing.

Table 4 shows the quantitative comparison results of the five object detection algorithms for UCAS-AOD. In Table 4, the AP values of airplane and car are 0.9488 and 0.8996 , respectively, in the object detection results using CNN-AOOF. The AP values of two kinds of objects using CNN-AOOF are greater than those of other object detection algorithms. Moreover, the mAP value of CNN-AOOF is the largest among the five object detection 
algorithms. The experimental results show that CNN-AOOF is superior to the other four object detection algorithms for UCAS-AOD.

Table 4. Performance comparisons of the five object detection algorithms in terms of AP values for UCAS-AOD dataset.

\begin{tabular}{cccc}
\hline & Airplane & Car & mAP \\
\hline Faster-RCNN [16] & 0.9270 & 0.7582 & 0.8426 \\
CNN-SOSF [33] & 0.9339 & 0.7965 & 0.8652 \\
YOLOv2 [20] & 0.7426 & 0.1501 & 0.4463 \\
YOLOv3 [21] & 0.9414 & 0.8805 & 0.9109 \\
CNN-AOOF & 0.9488 & 0.8996 & 0.9242 \\
\hline
\end{tabular}

Figure $9 \mathrm{a}, \mathrm{b}$ are the PRCs of the five object detection algorithms for airplane and car in UCAS-AOD, respectively. In Figure 9, we can see that for airplane and car, the PRC areas of CNN-AOOF are greater than those of the other object detection algorithms. The experimental results show that $\mathrm{CNN}$-AOOF outperforms the other object detection algorithms, and can obtain more accurate airplane and car detection results for UCAS-AOD dataset.

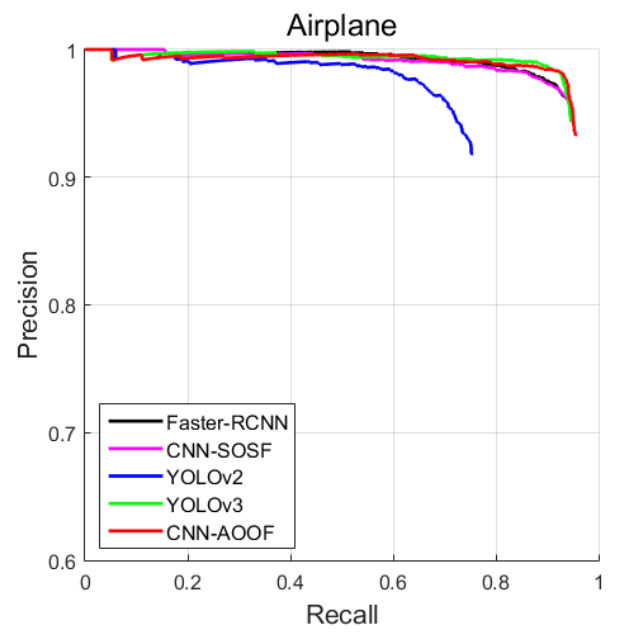

(a)

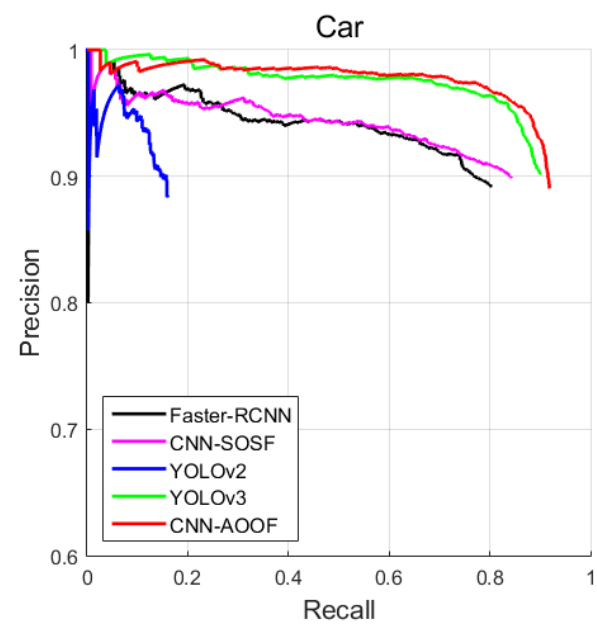

(b)

Figure 9. PRCs of the five object detection algorithms for (a) airplane, and (b) car in the UCAS-AOD dataset.

\subsection{Object Detection for HRSC2016}

HRSC2016 [46] dataset is used to compare CNN-AOOF with the other object detection algorithms (Faster-RCNN, CNN-SOSF, YOLOv2 and YOLOv3) to verify the object detection effectiveness of CNN-AOOF. HSRC2016 is a ship detection dataset of HSRI. Ship samples are labeled using OBB. Images are cropped from Google Earth. The size of images ranges from 300 pixels $\times 300$ pixels to 1500 pixels $\times 900$ pixels. There are 1061 images in HSRC2016, including 436 images for training, 181 images for validation, and 444 images for testing.

Table 5 shows the quantitative comparison results of the five object detection algorithms for HSRC2016. In Table 5, the AP values of the ship in the object detection results of the five algorithms are $0.8349,0.8301,0.423,0.8144$ and 0.8567 , respectively. The AP value of the ship using CNN-AOOF is greater than that of the other four object detection algorithms. The experimental results show that CNN-AOOF outperforms the other four object detection algorithms for HRSC2016. 
Table 5. Performance comparisons of the five object detection algorithms in terms of AP values for the HSRC2016 dataset.

\begin{tabular}{cc}
\hline & Ship \\
\hline Faster-RCNN [16] & 0.8349 \\
CNN-SOSF [33] & 0.8301 \\
YOLOv2 [20] & 0.4230 \\
YOLOv3 [21] & 0.8144 \\
CNN-AOOF & 0.8567 \\
\hline
\end{tabular}

Figure 10 is the PRC of five object detection algorithms for ship in HSRC2016. In Figure 10, we can see that for ship, the PRC area of CNN-AOOF is greater than that of the other four object detection algorithms. The experimental results show that $\mathrm{CNN}-\mathrm{AOOF}$ is superior to Faster-RCNN, CNN-SOSF, YOLOv2 and YOLOv3, and can obtain more accurate ship detection results for the UCAS-AOD dataset.

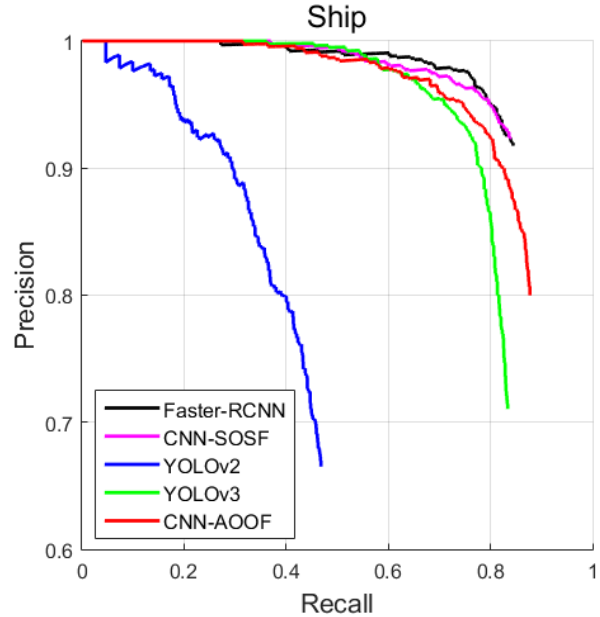

Figure 10. PRC of the five object detection algorithms for ship in HSRC2016 dataset.

\subsection{Object Detection for DOTA}

DOTA [40] is a multi-category object detection dataset for HSRIs. There are $2806 \mathrm{im}$ ages in the dataset. The training set, validation set and test set account for 1/3,1/6 and $1 / 2$ of the data set, respectively. The images range from about 800 pixels $\times 800$ pixels to 4000 pixels $\times 4000$ pixels. There are 15 kinds of objects (plane (PL), ship (SH), storage-tank (ST), baseball diamond (BD), tennis court (TC), basketball court (BC), ground track field (GTF), harbor (HA), bridge (BR), large vehicle (LV), small vehicle (SV), helicopter (HC), roundabout (RA), soccer ball field (SBF) and swimming pool (SP)) in the dataset. In DOTA, object samples are labeled using OBB.

Table 6 shows the quantitative comparison results of $\mathrm{CNN}-\mathrm{AOOF}$ and some state-ofthe-art object detection algorithms (RoI Trans, SCRDet, Li et al., Mask OBB). In Table 6, the mAP values of five object detection algorithms are $0.6956,0.7261,0.7328,0.7533$ and 0.7571 , respectively. The $\mathrm{mAP}$ of CNN-SOSF is 0.7571 , which is the largest value among the five object detection algorithms. The experimental results show that CNN-SOSF outperforms the other four object detection algorithms, and can obtain better object detection results for the DOTA dataset. 
Table 6. The quantitative comparison results of different object detection algorithms for the DOTA dataset.

\begin{tabular}{|c|c|c|c|c|c|c|c|c|c|c|c|c|c|c|c|c|}
\hline & PL & SH & ST & BD & TC & BC & GTF & HA & BR & LV & sv & $\mathrm{HC}$ & RA & SBF & SP & $\mathrm{mAP}$ \\
\hline RoI Trans [36] & 0.8864 & 0.8359 & 0.8146 & 0.7852 & 0.9074 & 0.7727 & 0.7592 & 0.6283 & 0.4344 & 0.7368 & 0.6881 & 0.4767 & 0.5354 & 0.5839 & 0.5893 & 0.6956 \\
\hline SCRDet [37] & 0.8998 & 0.7241 & 0.8686 & 0.8065 & 0.9085 & 0.8794 & 0.6836 & 0.6625 & 0.5209 & 0.6032 & 0.6836 & 0.6521 & 0.6668 & 0.6502 & 0.6824 & 0.7261 \\
\hline Li et al. [38] & 0.9021 & 0.7956 & 0.8468 & 0.7958 & 0.9083 & 0.834 & 0.7641 & 0.7417 & 0.4549 & 0.6827 & 0.7318 & 0.6486 & 0.6542 & 0.534 & 0.6969 & 0.7328 \\
\hline Mask OBB [39] & 0.8956 & 0.8563 & 0.8648 & 0.8595 & 0.8985 & 0.8381 & 0.729 & 0.7394 & 0.5421 & 0.7416 & 0.7652 & 0.6332 & 0.6964 & 0.5489 & 0.6906 & 0.7533 \\
\hline CNN-AOOF & 0.8821 & 0.7763 & 0.8612 & 0.8162 & 0.8954 & 0.8531 & 0.7293 & 0.8063 & 0.588 & 0.7882 & 0.7102 & 0.6361 & 0.6092 & 0.6263 & 0.7784 & 0.7571 \\
\hline
\end{tabular}

\section{Discussion}

In this section, CNN-AOOF is compared with other object detection algorithms by using visual evaluation. In two-stage object detectors, the object detection accuracy of CNN-SOSF is better than that of Faster-RCNN for WHU-RSONE, UCAS-AOD and HSRC2016 datasets. In single-stage object detectors, the object detection accuracy of YOLOv3 is better than that of YOLOv2. Therefore, CNN-AOOF is compared with CNNSOSF and YOLOv3 which have greater detection accuracy by using visual discrimination. Figure 11 shows some object detection result samples of CNN-SOSF, YOLOv3 and CNNAOOF for WHU-RSONE, UCAS-AOD and HSRC2016 datasets. Figure 11a-c are object detection results of CNN-SOSF, YOLOv3 and CNN-AOOF, respectively.
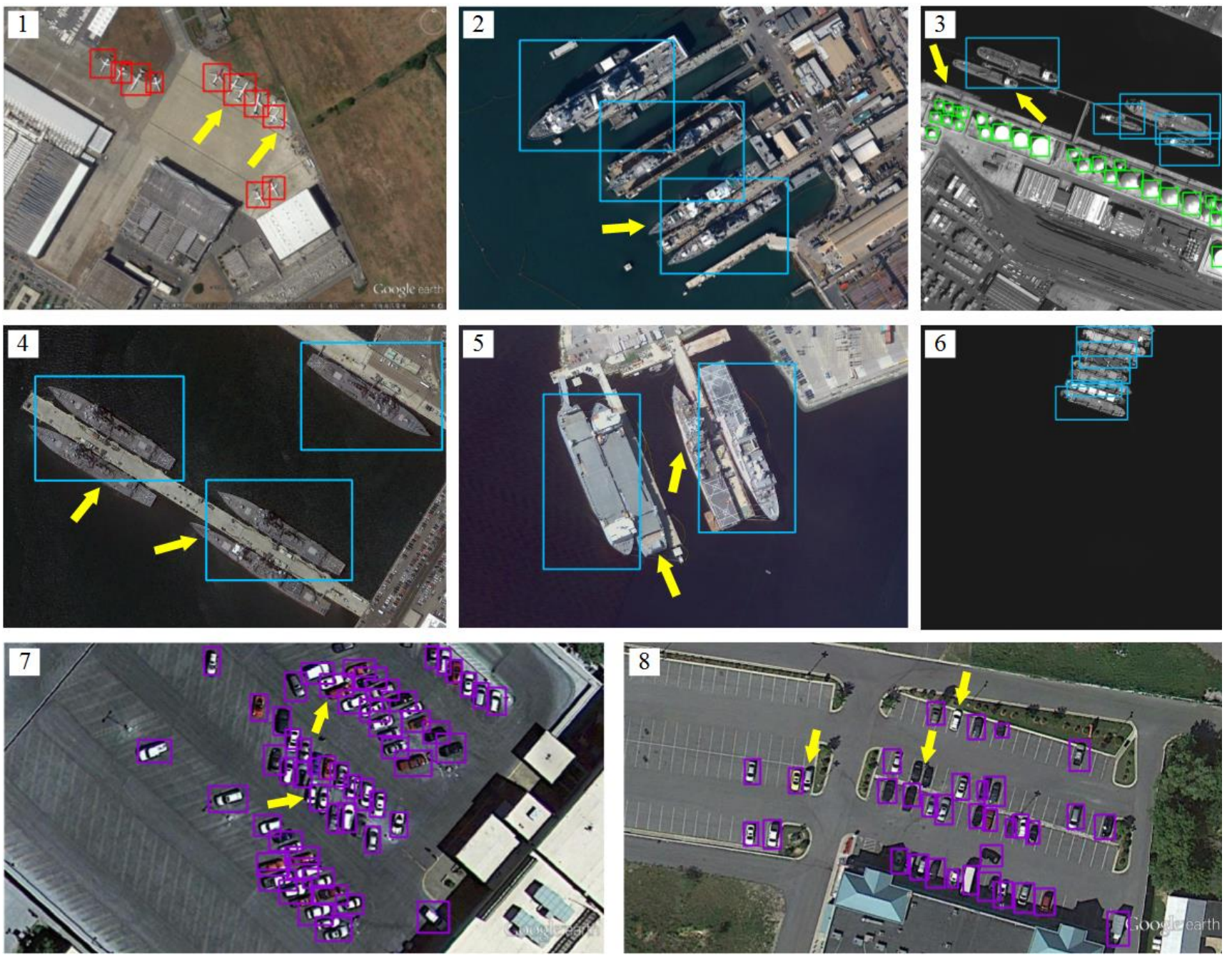

(a)

Figure 11. Cont. 

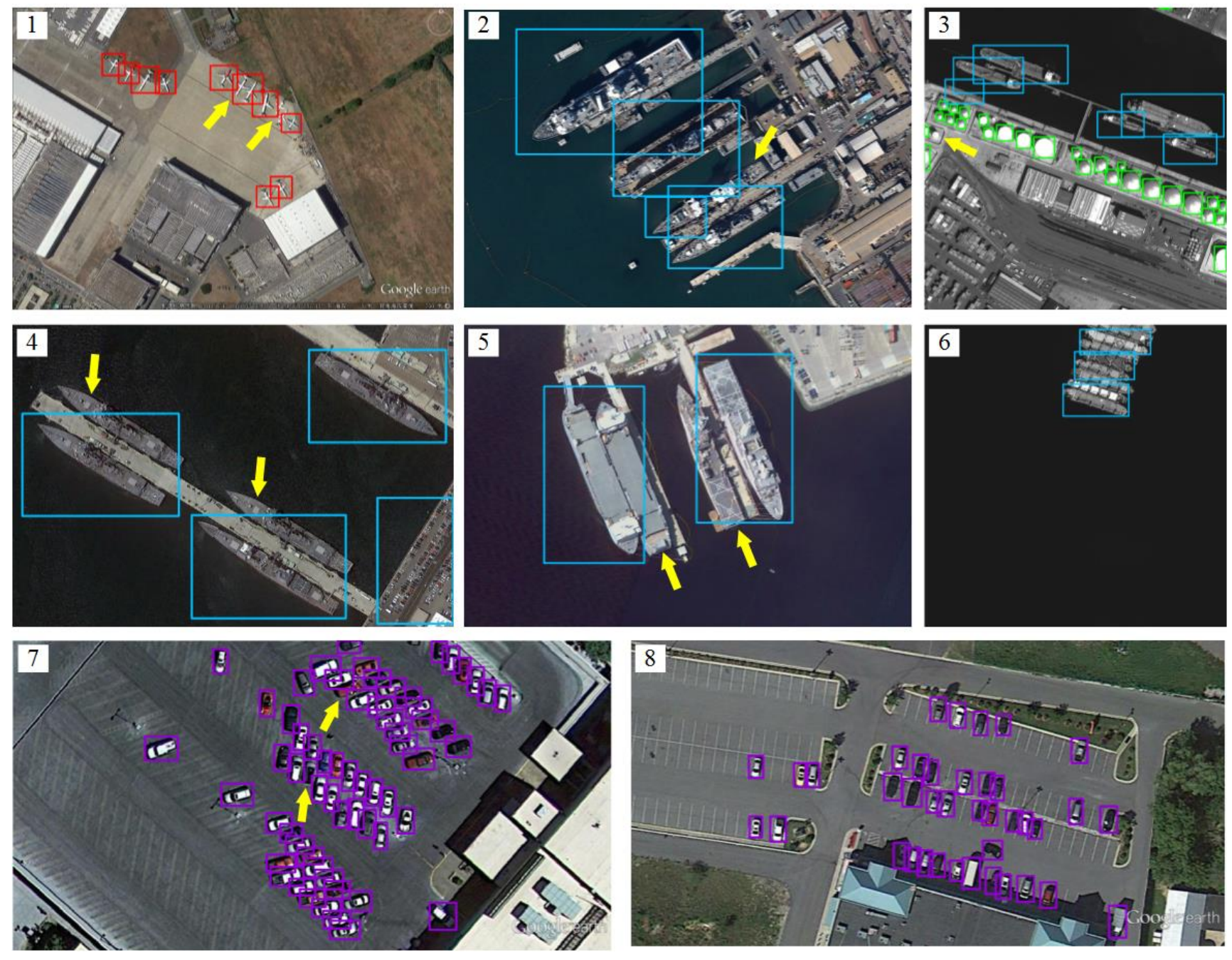

(b)

Figure 11. Cont. 

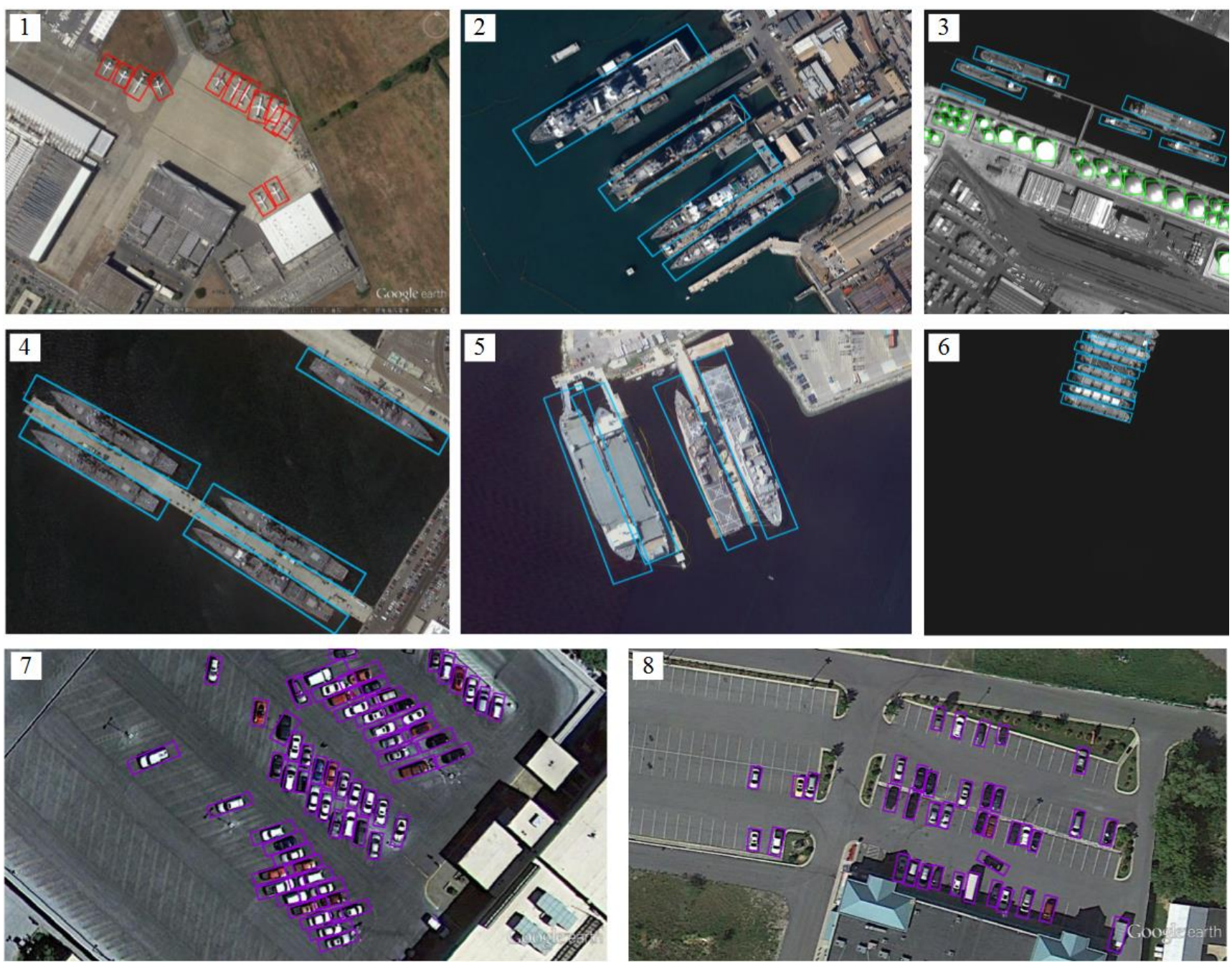

(c)

Figure 11. $(\mathbf{a}-\mathbf{c})$ are object detection results of CNN-SOSF, YOLOv3 and CNN-AOOF, respectively.

In Figure 11(a1,b1), due to the dense distribution of the airplanes, the airplanes indicated by the yellow arrow cannot be detected correctly using CNN-SOSF and YOLOv3. In Figure 11(c1), airplanes are correctly detected using CNN-AOOF.

In Figure 11(a2,b2), due to the ship with large aspect ratios, the ship indicated by the yellow arrow cannot be detected correctly using CNN-SOSF and YOLOv3. There are large redundant areas in the detection results of other ships. In Figure 11(c2), ships are correctly detected using $\mathrm{CNN}-\mathrm{AOOF}$.

In Figure 11(a3), due to the large aspect ratios and dense distribution of the ships, the ships indicated by the yellow arrow cannot be detected correctly using CNN-SOSF. In Figure 11(b3), the storage-tank indicated by the yellow arrow cannot be detected correctly using YOLOv3. In Figure 11(c3), ships and storage-tanks are correctly detected using CNN-AOOF.

In Figure 11(a4,b4), due to the ships with large aspect ratios, the ships indicated by the yellow arrow cannot be detected correctly using CNN-SOSF and YOLOv3. There are large redundant areas in the detection results of other ships. In Figure 11(c4), ships are correctly detected using $\mathrm{CNN}-\mathrm{AOOF}$.

In Figure 11(a5,b5), due to the ships with large aspect ratios, the ships indicated by the yellow arrow cannot be detected correctly using CNN-SOSF and YOLOv3. There are large 
redundant areas in the detection results of other ships. In Figure 11(c5), ships are correctly detected using $\mathrm{CNN}-\mathrm{AOOF}$.

In Figure 11(a6,b6), dense ships cannot be detected accurately using CNN-SOSF and YOLOv3. In Figure 11(c6), dense ships are detected accurately using CNN-AOOF.

In Figure 11(a7,b7), due to the dense distribution of cars, the cars indicated by the yellow arrow cannot be detected accurately using CNN-SOSF and YOLOv3. In Figure 11(c7), dense cars are detected accurately using CNN-AOOF.

In Figure 11(a8), due to the dense distribution of cars, the cars indicated by the yellow arrow cannot be detected accurately using CNN-SOSF. In Figure 11(b8,c8), cars are detected accurately using $\mathrm{CNN}-\mathrm{AOOF}$.

The experimental results show that CNN-SOSF and YOLOv3 are difficult to accurately detect objects with large aspect ratios and densely distributed objects because they use horizontal bounding boxes to detect objects. However, CNN-AOOF uses OBB to detect objects, and can accurately detect objects with large aspect ratios and densely distributed objects. Therefore, CNN-AOOF is superior to CNN-SOSF and YOLOv3 for WHU-RSONEOBB, UCAS-AOD and HSRC2016 datasets.

\section{Conclusions and Future Work}

With respect to the multi-angle features of object orientation in HSRIs object detection, a novel HSRIs object detection method based on convolutional neural networks with adaptive object orientation features $(\mathrm{CNN}-\mathrm{AOOF})$ is proposed in this paper. First, an adaptive object orientation regression method is proposed to obtain object regions in any direction. Then, a CNN framework for object detection of HSRIs is designed using the adaptive object orientation regression method. To verify the object detection effectiveness of CNN-AOOF, WHU-RSONE-OBB, UCAS-AOD, HSRC2016, and DOTA datasets are used to qualitatively and quantitatively compare $\mathrm{CNN}-\mathrm{AOOF}$ with some state-of-the-art object detection algorithms. The experimental results show that CNN-SOSF is superior to other state-of-the-art object detection algorithms, and can accurately detect objects with large aspect ratios and densely distributed objects for different object detection datasets of HSRIs. Object anchor scales are the vital factor affecting object detection results of HSRIs. In future work, how to adaptively adjust object anchor scales in the proposed method for different object detection tasks will be studied to obtain more accurate object detection results.

Author Contributions: Methodology, Software, Writing—original draft, Writing—review and editing, Z.D. Funding acquisition, Supervision, Writing-review and editing, M.W. Writing-review and editing, Y.W. and Y.L. Data collection, Y.F. and W.X. All authors have read and agreed to the published version of the manuscript.

Funding: This work was supported by the National Natural Science Foundation of China under Grant [No. 61825103 and 41871381]; the Fundamental Research Funds for the Central Universities under Grant [No. 2042021kf1030]; and the Key Laboratory of Ocean Geomatics, Ministry of Natural Resources, China under Grant [No. 2021A01].

Institutional Review Board Statement: Not applicable.

Informed Consent Statement: Not applicable.

Data Availability Statement: WHU-RSONE-OBB, UCAS-AOD, HSRC2016 and DOTA can be downloaded at https:/ / pan.baidu.com/s/1_Gdeedwo9dcEJqIh4eHHMA (accessed on 1 January 2022) (password: 1234), https:/ / github.com/fireae/UCAS-AOD-benchmark (accessed on 1 January 2022), https:/ / sites.google.com/site/hrsc2016/ (accessed on 1 January 2022) and https:/ / captain-whu. github.io/DOTA/index.html (accessed on 1 January 2022), respectively.

Acknowledgments: The authors would like to thank the editors and anonymous reviewers for their valuable comments, which helped improve this paper.

Conflicts of Interest: We declare that we have no conflict of interest. 


\begin{tabular}{ll}
\multicolumn{2}{l}{ Abbreviations } \\
HSRIs & High Spatial Resolution Remote Sensing Images \\
CNN & Convolutional Neural Networks \\
SVM & Support Vector Machines \\
HOG & Histograms of Oriented Gradients \\
R-CNN & Regional Convolutional Neural Networks \\
RPN & Region Proposal Network \\
CNN-SOSF & Convolutional Neural Networks with Suitable Object Scale Features \\
OBB & Oriented Bounding Box \\
CNN-AOOF & Convolutional Neural Networks with Adaptive Object Orientation Features \\
SSD & Single Shot Multibox Detector \\
YOLO & You Only Look Once \\
PRC & Precision-Recall Curve \\
IOU & Intersection-Over-Union \\
NMS & Non-Maximum Suppression \\
mAP & mean Average Precision
\end{tabular}

\section{References}

1. Li, D.; Wang, M.; Dong, Z.; Shen, X.; Shi, L. Earth observation brain (EOB): An intelligent earth observation system. Geo-Spatial Inf. Sci. 2017, 20, 134-140. [CrossRef]

2. Li, K.; Wan, G.; Cheng, G.; Meng, L.; Han, J. Object detection in optical remote sensing images: A survey and a new benchmark. ISPRS J. Photogramm. Remote Sens. 2020, 159, 296-307. [CrossRef]

3. Wang, Y.; Dong, Z.; Zhu, Y. Multiscale block fusion object detection method for large-scale high-resolution remote sensing imagery. IEEE Access 2019, 7, 99530-99539. [CrossRef]

4. Schilling, H.; Bulatov, D.; Niessner, R.; Middelmann, W.; Soergel, U. Detection of vehicles in multisensor data via multibranch convolutional neural networks. IEEE J. Sel. Topics Appl. Earth Observ. Remote Sens. 2018, 11, 4299-4316. [CrossRef]

5. Yang, X.; Sun, H.; Fu, K.; Yang, J.; Sun, X.; Yan, M.; Guo, Z. Automatic ship detection in remote sensing images from google earth of complex scenes based on multiscale rotation dense feature pyramid networks. Remote Sens. 2018, 10, 132. [CrossRef]

6. Wang, C.; Bai, X.; Wang, S.; Zhou, J.; Ren, P. Multiscale visual attention networks for object detection in vhr remote sensing images. IEEE Geosci. Remote Sens. Lett. 2019, 16, 310-314. [CrossRef]

7. Cheng, G.; Han, J. A survey on object detection in optical remote sensing images. ISPRS J. Photogramm. Remote Sens. 2016, 117, 11-28. [CrossRef]

8. Xiao, Z.; Liu, Q.; Tang, G.; Zhai, X. Elliptic fourier transformation-based histograms of oriented gradients for rotationally invariant object detection in remote-sensing images. Int. J. Remote Sens. 2015, 36, 618-644. [CrossRef]

9. Cheng, G.; Han, J.; Guo, L.; Qian, X.; Zhou, P.; Yao, X.; Hu, X. Object detection in remote sensing imagery using a discriminatively trained mixture model. ISPRS J. Photogramm. Remote Sens. 2018, 85, 32-43. [CrossRef]

10. Diao, W.; Sun, X.; Zheng, X.; Dou, F.; Wang, H.; Fu, K. Efficient saliency-based object detection in remote sensing images using deep belief networks. IEEE Trans. Geosci. Remote Sens. 2016, 13, 137-141. [CrossRef]

11. Han, J.; Zhou, P.; Zhang, D.; Cheng, G.; Guo, L.; Liu, Z.; Bu, S.; Wu, J. Efficient, simultaneous detection of multi-class geospatial targets based on visual saliency modeling and discriminative learning of sparse coding. ISPRS J. Photogramm. Remote Sens. 2014, 89, 37-48. [CrossRef]

12. Han, X.; Zhong, Y.; Zhang, L. An efficient and robust integrated geospatial object detection framework for high spatial resolution remote sensing imagery. Remote Sens. 2017, 9, 666. [CrossRef]

13. Girshick, R.; Donahue, J.; Darrell, T.; Malik, J. Region-based convolutional networks for accurate object detection and segmentation. IEEE Trans. Pattern Anal. Mach. Intell. 2016, 38, 142-158. [CrossRef] [PubMed]

14. He, K.; Zhang, X.; Ren, S.; Sun, J. Spatial pyramid pooling in deep convolutional networks for visual recognition. IEEE Trans. Pattern Anal. Mach. Intell. 2014, 37, 1904-1916. [CrossRef] [PubMed]

15. Girshick, R. Fast R-CNN. In Proceedings of the IEEE International Conference on Computer Vision (CVPR), Boston, MA, USA, 8-12 June 2015; pp. 1440-1448.

16. Ren, S.; He, K.; Girshick, R.; Sun, J. Faster R-CNN: Towards real-time object detection with region proposal networks. IEEE Trans. Pattern Anal. Mach. Intell. 2017, 39, 1137-1149. [CrossRef]

17. Redmon, J.; Divvala, S.; Girshick, R.; Farhadi, A. You only look once: Unified, real-time object detection. In Proceedings of the IEEE International Conference on Computer Vision and Pattern Recognition (CVPR), Las Vegas, NV, USA, 26 June-1 July 2016; pp. 779-788.

18. Liu, W.; Anguelov, D.; Erhan, D.; Szegedy, C.; Reed, S.; Fu, C.Y.; Berg, A.C. SSD: Single shot multibox detector. In Proceedings of the IEEE International Conference on Computer Vision and Pattern Recognition (CVPR), Las Vegas, NV, USA, 26 June-1 July 2016; pp. 21-37. 
19. Dai, J.; Li, Y.; He, K.; Sun, J. R-FCN: Object detection via region based fully convolutional networks. In Proceedings of the Neural Information Processing Systems (NIPS), Barcelona, Spain, 4-9 December 2016; pp. 379-387.

20. Redmon, J.; Farhadi, A. YOLO9000: Better, faster, stronger. In Proceedings of the IEEE International Conference on Computer Vision and Pattern Recognition (CVPR), Honolulu, HI, USA, 21-26 July 2017; pp. 6517-6525.

21. Redmon, J.; Farhadi, A. YOLOv3: An incremental improvement. arXiv 2018, arXiv:1804.02767.

22. Wen, N.; Guo, R.; Ma, D.; Ye, X.; He, B. AIoU: Adaptive bounding box regression for accurate oriented object detection. Int. J. Intell. Syst. 2022, 37, 748-769. [CrossRef]

23. Zhang, Y.; Fu, K.; Sun, H.; Sun, X.; Zheng, X.; Wang, H. A multi-model ensemble method based on convolutional neural networks for aircraft detection in large remote sensing images. Remote Sens. Lett. 2018, 9, 11-20. [CrossRef]

24. Cheng, G.; Zhou, P.; Han, J. Learning rotation-invariant convolutional neural networks for object detection in vhr optical remote sensing images. IEEE Trans. Geosci. Remote Sens. 2016, 54, 7405-7415. [CrossRef]

25. Wu, X.; Hong, D.; Tian, J.; Chanussot, J.; LI, W.; Tao, R. Orsim detector: A novel object detection framework in optical remote sensing imagery using spatial-frequency channel features. IEEE Trans. Geosci. Remote Sens. 2019, 57, 5146-5158. [CrossRef]

26. Ren, Y.; Zhu, C.; Xiao, S. Small object detection in optical remote sensing images via modified faster R-CNN. Appl. Sci. 2018, 8 , 813. [CrossRef]

27. Yang, C.; Li, W.; Lin, Z. Vehicle object detection in remote sensing imagery based on multi-perspective convolutional neural network. ISPRS Int. J. Geo-Inf. 2018, 7, 249. [CrossRef]

28. Long, Y.; Gong, Y.; Xiao, Z.; Liu, Q. Accurate object localization in remote sensing images based on convolutional neural networks. IEEE Trans. Geosci. Remote Sens. 2017, 55, 2486-2498. [CrossRef]

29. Guo, W.; Yang, W.; Zhang, H.; Hua, G. Geospatial object detection in high resolution satellite images based on multi-scale convolutional neural network. Remote Sens. 2018, 10, 131. [CrossRef]

30. Chen, Z.; Zhang, T.; Ouyang, C. End-to-end airplane detection using transfer learning in remote sensing images. Remote Sens. 2018, 10, 139. [CrossRef]

31. Li, K.; Cheng, G.; Bu, S.; You, X. Rotation-insensitive and context augmented object detection in remote sensing images. IEEE Trans. Geosci. Remote Sens. 2018, 56, 2337-2348. [CrossRef]

32. Deng, Z.; Sun, H.; Zhou, S.; Zhao, J.; Lei, L.; Zou, H. Multi-scale object detection in remote sensing imagery with convolutional neural networks. ISPRS J. Photogramm. Remote Sens. 2018, 145, 3-22. [CrossRef]

33. Dong, Z.; Wang, M.; Wang, Y.; Zhu, Y.; Zhang, Z. Object detection in high resolution remote sensing imagery based on convolutional neural networks with suitable object scale features. IEEE Trans. Geosci. Remote Sens. 2020, 58, 2104-2114. [CrossRef]

34. Liu, W.; Ma, L.; Wang, J.; Chen, H. Detection of multiclass objects in optical remote sensing images. IEEE Geosci. Remote Sens. Lett. 2019, 16, 791-795. [CrossRef]

35. Ma, H.; Liu, Y.; Ren, Y.; Yu, J. Detection of collapsed building in post-earthquake remote sensing images based on the improved YOLOv3. Remote Sens. 2019, 12, 44. [CrossRef]

36. Ding, J.; Xue, N.; Long, Y.; Xia, G.S.; Lu, Q. Learning RoI Transformer for Detecting Oriented Objects in Aerial Images. In Proceedings of the IEEE Conference on Computer Vision and Pattern Recognition, Los Angeles, CA, USA, 16-19 June 2019.

37. Li, C.; Xu, C.; Cui, Z.; Wang, D.; Zhang, T.; Yang, J. Feature-Attentioned Object Detection in Remote Sensing Imagery. In Proceedings of the IEEE International Conference on Image Processing, Taipei, Taiwan, 22-25 September 2019; pp. 3886-3890.

38. Yang, X.; Yang, J.; Yan, J.; Zhang, Y.; Zhang, T.; Guo, Z.; Sun, X.; Fu, K. SCRDet: Towards More Robust Detection for Small, Cluttered and Rotated Objects. In Proceedings of the IEEE International Conference on Computer Vision, Seoul, Korea, 27 October2 November 2019.

39. Wang, J.; Ding, J.; Guo, H.; Cheng, W.; Pan, T.; Yang, W. Mask OBB: A Semantic Attention-Based Mask Oriented Bounding Box Representation for Multi-Category Object Detection in Aerial Images. Remote Sens. 2019, 11, 2930. [CrossRef]

40. Xia, G.; Bai, X.; Ding, J.; Zhu, Z.; Belongie, S.; Luo, J.; Datcu, M.; Pelillo, M.; Zhang, L. Dota: A large-scale dataset for object detection in aerial images. In Proceedings of the IEEE International Conference on Computer Vision and Pattern Recognition (CVPR), Salt Lake City, UT, USA, 18-22 June 2018; pp. 3974-3983.

41. Qian, X.; Lin, S.; Cheng, G.; Yao, X.; Ren, H.; Wang, W. Object detection in remote sensing images based on improved bounding box regression and multi-level features fusion. Remote Sens. 2020, 12, 143. [CrossRef]

42. Henderson, P.; Ferrari, V. End-to-end training of object class detectors for mean average precision. In Proceedings of the Asian Conference on Computer Vision (ACCV), Kyoto, Japan, 30 November-4 December 2016; pp. 198-213.

43. Zhu, H.; Chen, X.; Dai, W.; Fu, K.; Ye, Q.; Jiao, J. Orientation robust object detection in aerial images using deep convolutional neural network. In Proceedings of the IEEE International Conference Image Processing, Quebec City, QC, Canada, 27-30 September 2015; pp. 3735-3739.

44. Qian, W.; Yang, X.; Peng, S.; Guo, Y.; Yan, J. Learning modulated loss for rotated object detection. arXiv 2019, arXiv:1911.08299.

45. Yang, X.; Yan, J.; Feng, Z.; He, T. R3Det: Refined single-stage detector with feature refinement for rotating object. In Proceedings of the IEEE International Conference on Computer Vision and Pattern Recognition (CVPR), Seattle, WA, USA, 14-19 June 2020.

46. Liu, Z.; Wang, H.; Weng, L.; Yang, Y. Ship rotated bounding box space for ship extraction from high-resolution optical satellite images with complex backgrounds. IEEE Geosci. Remote Sens. Lett. 2016, 13, 1074-1078. [CrossRef] 